\title{
ISLAMFEINDLICHKEIT - VOM GASTARBEITER ZUM MUSLIM
}

ISLAM DÜSMANLIḠI - KONUK IȘÇIDEN MÜSLÜMANA

ISLAMOPHOBIA (ISLAMFEINDLICHKEIT) - FROM GUEST WORKER TO MUSLIM

\section{NAIME CAKIR-MATTNER}

PROF. DR. GIESSEN JUSTUS-LIEBIG ÜNIVERSITESI, TARIH VE KÜLTÜR BILIMLERI BÖLÜMÜ, ISLAM ILAHIYATI ANABILIM DALI, GIESSEN, ALMANYA.

naime.cakir-mattner@islamtheologie.uni-giessen.de

(iD) http://orcid.org/0000-0002-3453-2780

doi http://dx.doi.org/10.46353/k7auifd.1002752

Makale Bilgisi / Article Information

Makale Türü / Article Types

Araștırma Makalesi / Research Article

Geliș Tarihi / Received

30 Eylül / September 2021

Kabul Tarihi / Accepted

27 Aralık / December 2021

Yayın Tarihi / Published

Aralık / December 2021

Yayın Sezonu / Pub Date Season

Aralık / December

Atıf / Cite as

Çakir-Mattner, Naime, "Islamfeindlichkeit - vom Gastarbeiter zum Muslim [i̇slam Düșmanlı̄̆ı Konuk İșçiden Müslümana]". Kilis 7 Aralık Üniversitesi İlahiyat Fakültesi Dergisi - Journal of the Faculty of Theology 8/2 (Aralık/December 2021): 417-429

Intihal / Plagiarism: Bu makale, en az iki hakem tarafından incelendi ve intihal içermediḡi teyit edildi. / This article has been reviewed by at least two referees and scanned via a plagiarism software.

Published by Kilis 7 Aralık Üniversitesi, Illahiyat Fakültesi - Kilis 7 Aralık University, Faculty of Theology, Kilis, 79000 Turkey. 


\section{ISLAMFEINDLICHKEIT - VOM GASTARBEITER ZUM MUSLIMํำ}

\section{Zusammenfassung}

Zunächst wird im vorliegenden Artikel in historischer Rückschau darauf hingewiesen, dass in vielen europäischen Ländern sich das als bedrohlich empfundene Fremde gegenüber dem vertrauten Eigenen wesentlich entlang der islamischen Religionszugehörigkeit zeigt. Diese damit zunehmende „Islamfeindlichkeit“ wird, was die terminologische Unsicherheit kennzeichnet, mit unterschiedlichen Begriffen bezeichnet wie Muslimfeindlichkeit, Islamophobie bzw. antimuslimischen Rassismus. Die Autorin zeigt im Weiteren auf, dass dieses Fremde insbesondere infolge der Entkolonialisierung in den 1950er- und 1960er-Jahren und der dadurch ausgelösten post-kolonialen Wanderungen aus Nordafrika und durch die Gastarbeiterwelle der „Pioniermigranten“ aus muslimisch geprägten Ländern nach Europa zunehmend ins Blickfeld der Öffentlichkeit geriet. Mit der sichtbaren Zunahme der religiösen Orientierung und den damit verbundenen Riten und Symbole wuchs offenkundig die Skepsis gegenüber dieser aus der Fremde importierten Religion, die mittlerweile in den europäischen Metropolen angekommen ist. Hier entwickelte sich sowohl durch die Bleibeorientierung der ehemaligen Einwanderer als auch durch die damit verbundene Loslösung des Islam aus seinen ursprünglichen Kernländern (Entterritorialisierung) das Kennzeichen eines neuen sichtbar gewordenen „europäischen Islam“, der die Identitätskonstruktionen des „Eigenen“ insgesamt zu erschüttern scheint. Dies ist der Hintergrund dessen, dass diese Entwicklung eine massive Abwehr bis hin zur Feindlichkeit gegenüber dem Islam auszulösen scheint. In diesem Spannungsfeld versuchen die neuen „europäischen Muslime“, öffentliche Räume religiös zu besetzen und einen eigenen Weg zwischen Moderne und Tradition zu finden. In diesem Prozess geht es dieser Generation in weiten Teilen längst nicht mehr um eine bloße Konservierung des vom jeweiligen Herkunftsland importierten elterlichen Religionsverständnisses, sondern sie sind vielmehr im Sinne einer relativen Entkoppelung des Islam von der Herkunftskultur darum bemüht, im Rückgriff auf die Primärquellen des Islam ein enttraditionalisiertes, gewissermaßen "reines“ Islamverständnis zu entwickeln und zu rekonstruieren und diesen Islam öffentlich sichtbar und lebbar zu machen. Insofern findet mit diesem Bemühen um ein neues Religionsverständnis nicht nur eine Entterritorialisierung des Islam statt, sondern auch eine Form der Enttraditionalisierung, bei der es unter anderem darum geht, den Islam in die europäische Kultur zu implementieren.

Dieser Prozess der Integration löste Abwehrreaktionen aus, wodurch der Islam nicht nur zum zentralen Differenzmarker zwischen dem Eigenen und dem Fremden wurde, sondern zunehmend als feindlicher Antagonist zum Eigenen erlebt wird. In diesem Zusammenhang wird durch die Autorin verdeutlicht, dass im deutschen Kontext sich dieser Prozess im Zuge der Integrationsbemühungen der Nachkommen der ehemaligen Gastarbeitergeneration aufzeigen lässt. Im Zuge von Bleibeorientierung und zunehmendem Selbstbewusstsein der zweiten und der dritten Generation wurden die etablierten Rollen des Gastgebers und des Gastes infrage gestellt, weshalb es einer Neujustierung der Rollen innerhalb des Gesellschaftsgefüges bedurfte, die die Marginalisierung der mittlerweile etablierten Einwanderer weiterhin zu gewährleisten hatte.

In ihrem Artikel führt Naime Çakır-Mattner somit kurz in die moderne Form der Islamfeindlichkeit ein, die sie auch als eine Reaktion auf die Dauerpräsenz von

Dieser Artikel wurde bis auf wenige Änderungen bereits schon publiziert (vgl. Naime Çakır, „Das Eigene und das Fremde - zwischen Heterophobie und Rassismus", In: Bülent Ucar und Wassilis Kassis (Hrsg.), Antimuslimischer Rassismus und Islamfeindlichkeit in Deutschland und Europa (Göttingen: Vandenhoeck \& Ruprecht 2019), 77-101. 
Muslim_innen im Zuge der Einwanderungsgeschichte in Deutschland analysiert. Hierbei wird der Islam zum Gegenstand öffentlicher Anerkennungskonflikte. Im Konkurrenz um die besten Plätze innerhalb der Gesellschaft werden antiislamische Vorurteile und Feindbilder aktiviert und als Ausschließungspraxis eingesetzt, um insbesondere diejenigen, die den sozialen Aufstieg vollzogen haben, an die randständigen Positionen innerhalb der Gesellschaft zu binden. In diesem Zusammenhang verweist die Autorin auf mehrere rechts-populistische Gruppierungen, die neben einer mittlerweile rechts orientierten etablierten Partei (AfD) entstanden und im gemeinsamen Bestreben vereint sind, der Islamisierung im eigenen Land Einhalt zu bieten, um das durch Islamisierung bedrohte Verschwinden einer europäischekulturellen Identität zu verhindern.

Schlüsselbegriffe: Islam, Muslime, Integration, Migration, Rassismus, Islamophobie, Islamfeindlichkeit

\title{
ISLAMOPHOBIA (ISLAMFEINDLICHKEIT) - FROM GUEST WORKER TO MUSLIM
}

\begin{abstract}
The article points out, in historical retrospect, that in many European countries the foreign, which is perceived as threatening in comparison to the familiar, is essentially shown along the lines of Islamic religious affiliation. This increasing "Islamophobia", which is characterised by terminological uncertainty, is described with different terms such as Muslimphobia, Islamophobia or anti-Muslim racism. In addition, the author shows that this foreignness increasingly came into the public eye, particularly because of decolonisation in the 1950s and 1960s associated with the post-colonial migrations from North Africa, and through the wave of guest workers of "pioneer migrants" from Muslim-majority countries to Europe. With the visible increase in religious orientation associated with the rites and symbols, there was an obvious growth in scepticism towards this religion, which is imported from abroad and has now arrived in the European metropolises. Here, the characteristic of a new visible "European Islam" developed through the former immigrants' orientation towards staying and through the associated disengagement of Islam from its original core countries (de-territorialisation), which seems to shake the identity constructions of the "own" as a whole. This development seems to trigger a massive rejection of Islam, even hostility towards it. Within this field of tension, the new "European Muslims" are trying to fill public spaces religiously and to find their own way between modernity and tradition. This new generation of Muslims has long since stopped to be concerned with merely preserving the parental understanding of religion imported from the respective country of origin. In the sense of a decoupling of Islam from the culture of origin, they are striving to develop and reconstruct a de-traditionalised, to some extent "pure" understanding of Islam, by taking recourse to the primary sources of Islam, and to make this Islam visible and liveable in public. In this respect, this effort towards a new understanding of religion is not only a deterritorialisation of Islam, but also a form of de-traditionalization, which, among other things, is about implementing Islam in European culture.

This process of integration triggered defensive reactions, whereby Islam not only became the central marker of difference between its own and the foreign, increasingly began to be perceived as a hostile opponent for them. In this context, the author makes clear that this process can be seen in the German context during the integration efforts of the descendants of the former guest worker generation. In the
\end{abstract}


course of the second and third generation's orientation towards a long-term home and increasing self-confidence, the established roles of host and guest were challenged, which is why a readjustment of the roles within the social structure was required, to ensure the marginalisation of the by now established immigrants.

In her article, Naime Çakır-Mattner thus briefly introduces the modern form of Islamophobia, which she also analyses as a reaction to the permanent presence of Muslims during immigration history in Germany. In this process, Islam becomes the object of public conflicts of recognition. In the competition for the best places within society, anti-Islamic prejudices and images of the enemy are activated and used as a practice of exclusion in order to bind especially those who have achieved social advancement to the marginal positions within society. In this regard, the author refers to several right-wing populist groups that have emerged alongside a now right-wing established party (AfD) and are united in the common endeavour to put a stop to Islamisation in their own country to prevent the disappearance of a European cultural identity threatened by Islamisation.

Keywords: Islam, Muslims, Integration, Migration, Racism, Islamophobia

\section{İSLAM DÜŞMANLIĞI - KONUK İŞÇİDEN MÜSLÜMANA}

\section{Öz}

$\mathrm{Bu}$ makalede geriye dönük tarihi bir bakışla Avrupa'ının aşina olduğu kendi benliği karşısında Avrupadaki birçok ülkede tehdit olarak algılanan yabancılığın İslami din mensubiyeti çizgisinde gösterdiği belirtilmektedir. Böylece artan "İslam düşmanlığı", terminolojik tedirginliği gösterdiği şekliyle örneğin Müslüman düşmanlığı, İslamofobi veya Müslüman aleyhtarlı ırkçlık gibi farklı kavramlarla isimlendirilmektedir.

Makalenin sonraki kısımlarında yazar özellikle 1950 ve 1960’lı yıllarda misafir işçi dalgasının "göçmenlerin öncülerinin" Müslüman kültürünün etkin olduğu ülkelerden Avrupa’ya göç, Avrupa kamuoyunun dikkatini çekmeye başladığı üzerinde durmaktadır. Dini oryantasyon ile buna bağlı olan dini törenler ve sembollerin artarak görünür hale gelmesi ile artık Avrupa metropollerine ulaşmış olan ve yabancı ülkelerden ithal edilen bu dine karşı tedirginlik aleni hale gelmiştir. Bunlar nezdinde ise hem önceki göçmenlerin ülkede yerleşik şekilde kalma oryantasyonları hem de bununla bağlantılı olarak İslam’ın asıl menşe ülkelerinden bağlarını koparması (Enttoritarialisierung) ile "Avrupâî İslam’ın" görünür hale gelmiş olması, "Avrupa’lı insanların kendi benliklerinin” şahsiyet inşalarını bütüncül olarak sarsmış gibi görünmektedir.

Bu gelişme, İslam’ın müthiş bir dirençten düşmanlığa kadar varan bir hareketi tetiklediği intibaını vermesinin arkaplanıdır. Bu gerginlik alanında yeni "Avrupâî Müslümanlar" kamusal alanları dini olarak simgelemeye ve modernite ile gelenek arasında kendi yollarını bulmaya çalışmaktadırlar. Bu kuşak açısından bu süreç artık geniş kapsamda ilgili menşe ülkeden ebeveynin din anlayışının ithali sözkonusu değil, bilakis bu kuşak İslam’ın menşe kültürlerden görece bağımlılığını koparma anlamında İslam’ın ana kaynaklarına dayanarak gelenekten arındırılmış, bir anlamda "temiz" bir İslam anlayışı geliştirme ve yeniden inşa ile bu İslam’ı kamu nezdinde görünür ve yaşanır hale getirmek için gayrette bulunmaktadırlar. Bu yeni din anlayışına bağlı gayretler bağlamında İslam’ın Avrupa kültürüne uyarlama açısından sadece İslam’ın bir ülkeden bağının koparılmasının (Entteritorialisierung) gerçekleşmesiyle kalmamakta, bilakis aynı zamanda gelenekten de bağımsızlaşmanın bir şekli de gerçekleşmektedir.

Uyumun bu süreci, bazı savunma reaksiyonlarına sebep olarak İslam, bu yolla kendi benliği ile yabancılar arasında sadece farkların markörü olmakla kalmamış, bilakis gitgide kendileri için düşman bir muhalif olarak algılanmaya başlamıştır. Bu çerçe- 
vede Almanya bağlamında bu sürecin eski misafir işçi neslinin sonraki kuşaklarının uyum süreci gayretleri üzerinde gösterilebildiği etki üzerinde durulmaktadır. Almanya'da kalıcı hale gelme oryantasyonu ile ikinci ve üçüncü kuşağın artan özgüvenleri ile yerleşik ev sahibi ve misafir rollerinin sorgulanır hale geldimesinden dolayı toplum dokusundaki rollerin yeniden düzenlenmesi gerektiği ve bu hususun da bu arada yerleşik hale gelmiş göçmenlerin artarak marjinalleşmelerini beraberinde getirmesi sözkonusudur.

Naime Çakır-Mattner makalesinde böylece İslam düşmanlığının modern bir şeklini özetlemekte ve Almanyadaki göç tarihi bağlamında bunu Müslümanların daimi mevcudiyetine bir tepki olarak da analiz etmektedir. Bu bağlamda ise İslam kamusal tanınmaya yönelik çatışmaların konusu haline gelmektedir. Toplum nezdindeki en iyi pozisyon ve yerlerle ilgili gelişen rekabet kapsamında sosyal tırmanışını gerçekleştiren kişileri toplumda bulunan çeperlerdeki ve kenarlardaki pozisyonlara bağlama şekliyle İslam aleyhtarı önyargılar ve düşman algılarının aktif hale getirilmesi ve dışlama uygulamaları sözkonusudur. Bu bağlamda yazar, birçok sağ oryantasyonu bulunan ve yerleşik populist grupların (AfD) yanında birçok sağ populist grupların ortak gayrette birleşerek kendi ülkelerindeki İslamlaşmayı engellemeye yönelik gayretler göstererek İslamlaşma ile Avrupâî kültürel kimliğin ortadan kalkması tehdine karşı bir girişim olduğuna işaret etmektedir.

Anahtar Kelimeler: İslam, Müslümanlar, Entegrasyon, Göç, Irkçılık, İslamofobi, İslam Düşmanlığı

\section{EINLEITUNG}

$\mathrm{n}$ der wissenschaftlichen wie in der öffentlichen Debatte um Is-
lamfeindlichkeit existiert eine gewisse terminologische Unsicher-
heit zur Bezeichnung dieses Phänomens. Die auf antiislamische Vorurteile bezogenen Termini, die teilweise synonym benutzt werden, reichen von Islamfeindlichkeit, ${ }^{2}$ Muslimfeindlichkeit, ${ }^{3}$ Islamophobie bzw. islamophober Populismus ${ }^{4}$ bis hin zum antimuslimischen Rassismus ${ }^{5}$. Ganz allgemein umfasst Islamphobie die „Ablehnung und Angst vor Muslimen, ihrer Kultur sowie ihren öffentlichen, politischen und religiösen Aktivitäten.“6 Muslimfeindlichkeit zeigt sich in „negativ-stereotypen Hal-

2 vgl. Achim Bühl, Islamfeindlichkeit in Deutschland (Hamburg: VSA, 2010).; Thorsten Gerald Schneiders (Hrsg.), Islamfeindlichkeit, Wenn die Grenzen der Kritik verschwimmen (Wiesbaden: VS-Verlag, 2009).

3 vgl. Heiner Bielefeldt, „Facetten von Muslimfeindlichkeit. Differenzierung als Fairnessgebot.“ Überarbeitete Fassung eines Vortrags vom 6 (September 2010), 2. (http://www.deutsche-islam konfe-renz. de/SharedDocs/Anlagen/DE/DIK/Downloads/Sonstiges/vortrag-bielefeldt,templateId=raw,proper ty=publicationFile.pdf/vortrag-bielefeldt.pdf)

4 vgl. Wilhelm Heitmeyer (Hrsg.). Deutsche Zustände (Frankfurt/Main: Suhrkamp, 2002).; Wilhelm (Hrsg.), Deutsche Zustände (Frankfurt/Main: Suhrkamp, 2003).; Heitmeyer, Wilhelm (Hrsg.). Deutsche Zustände (Frankfurt/Main: Suhrkamp, 2008); Farid Hafez (Hrsg.), Jahrbuch für Islamophobieforschung (Wien: ZDB-Verlag 2010), 16

5 vgl. Iman Attia, Die „westliche Kultur" und ihr Anderes. Zur Destruktion von Orientalismus und antimusli-mischem Rassismus (Bielefeld: transcript, 2009).

6 Wilheim Heitmeyer, „Gruppenbezogene Menschenfeindlichkeit - Die theoretische Konzeption und empirische Ergebnisse aus 2002, 2003 und 2004“, In: Wilhelm Heitmeyer, (Hrsg.), Deutsche Zustände (Frankfurt/Main: Suhrkamp 2005), 15 
tungen gegenüber dem Islam und seinen tatsächlichen oder mutmaßlichen

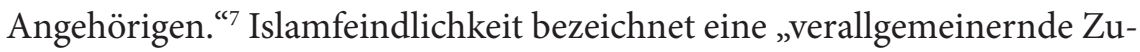
weisung (negativ-)stereotyper Fakten und Fiktionen auf den Islam und/ oder die Muslime, die man spontan dieser Gruppe zuordnet. “8

Diese teilweise synonym gebrauchte Terminologie ist sicherlich dem Wunsch einer inhaltlichen Differenzierung dieses Phänomens geschuldet, was sich offenbar als schwierig erweist. Insbesondere der gegenwärtig im wissenschaftlichen Diskurs etablierte Terminus „Islamophobie“ zur Kennzeichnung des Phänomens der Ausgrenzung und Diskriminierung einer Menschengruppe ist in mehrfacher Hinsicht problematisch, da ihm mit dem Suffix „Phobie“ eine psychopathologische und damit entlastende enthistorisierende Konnotation anhaftet. Der Begriff „Phobie“ bezieht sich im allgemeinen Sinne auf ein psychopathologisches Krankheitsphänomen wie Klaustro- oder Agoraphobie und wäre demnach Gegenstand einer psychotherapeutischen bzw. pharmakologischen Therapie. Demgegenüber beinhaltet eine "Islamophobie“ im Kontext islamfeindlicher Positionen negativ-ablehnende Einstellungen gegenüber Muslim_innen, die keinesfalls psycho-pathologisch erklärbar, geschweige denn entschuldbar sind, da es sich hierbei wohl kaum um psycho-pathologische Hass-Symptome mit Krankheitswert handelt, sondern wohl eher um sozial produzierte Haltungen gegenüber dem unerwünschten Anderen des Eigenen. ${ }^{9}$ Zentral zur Bezeichnung des Phänomens Islamfeindlichkeit sind demnach primär die inhaltlichen Aspekte wie eine vor-urteilende Zuweisung (negativ-)stereotyper Fakten und Fiktionen auf den Islam und auf Menschen, die man aus unterschiedlichen Gründen dieser Gruppe zuordnet.

Çakır spricht in diesem Zusammenhang im Kontext der modernen Islamfeindlichkeit vom antiislamischen Ethnizismus im Sinne des Rassismus ohne Rassen. Der antiislamische Ethnizismus „thematisiert das gesamte Spektrum negativer Einstellungen, das von diskriminierenden Vorurteilen bis hin zu offenen rassistischen Haltungen und Handlungen reicht, die von einer Ideologie der Ungleichwertigkeit mit impliziten unkorrigierbaren Vorurteilen und geschlossenen Feindbildern gekennzeichnet sind. “ 10

\footnotetext{
Heiner Bielefeldt, „Das Islambild in Deutschland. Zum öffentlichen Umgang mit der Angst vor dem Islam“, In: Th. G. Schneiders, (Hrsg.), Islamfeindlichkeit. Wenn die Grenzen der Kritik verschwimmen. (Wiesbaden: VS-Verlag, 2009), 182

8 Sabine Schiffer, „Islamophobie - Plädoyer für eine internationale Bezeichnung“, In. INAMO. Feindbild Islam. Islamfeindlichkeit und Rechtspopulismus, Heft 68, Jahrgang 17, Winter 2011, 22

9 vgl. Naime Çakır, Islamfeindlichkeit - Anatomie eines Feindbildes in Deutschland (Bielefeld: Transcript, 2014), $153 \mathrm{f}$.

${ }_{10}$ Naime Çakır, Islamfeindlichkeit, $151 \mathrm{f}$.
} 


\section{MODERNE FORM DER ISLAMFEINDLICHKEIT}

Antiislamische Motive und negative Bilder über den Islam können wir historisch in der mittelalterlichen Auseinandersetzung des Christentums mit dem Islam und später auch im Zuge des Orientalismus' im 19. Jahrhundert finden. Die Anfänge der „modernen“ Islamfeindlichkeit ist am ehesten im 20. Jahrhundert zu suchen. Allerdings sind der historische und die moderne Form der Islamfeindlichkeit nicht identisch, obgleich auch Gemeinsamkeiten zu finden sind. Beispielsweise rekurrierte die historische Islamfeindlichkeit auf religiös begründete Weltbilder, während die moderne Form der Islamfeindlichkeit antiislamische Vorurteile und Feindbilder als Mechanismen der sozialen Ausgrenzung gegenüber Migrant_innen nutzt, um insbesondere diejenigen, die bereits in der Mitte der Gesellschaft angekommen sind, an die randständigen Positionen innerhalb der Gesellschaft zu binden. War das historische Thema der Islamfeindlichkeit, den Fremden in der Fremde zu bekämpfen, ist das Thema der modernen Islamfeindlichkeit, den „Fremden“ im Inneren zu bekämpfen. Der Fremde im Inneren erscheint hierbei gefährlicher als der äußere Feind, der durch territoriale Grenzziehungen beherrschbar erscheint, während der Fremde im Inneren sich der Kontrolle jederzeit entziehen kann und somit unberechenbar bleibt. Die moderne Form der Islamfeindlichkeit ist auch eine Reaktion auf die Dauerpräsenz von Muslimen im Zuge der Einwanderungsgeschichte in Deutschland und die damit verbundene Irritation der Rollen zwischen dem Gast und dem Gastgeber. Die Gäste haben schon längst die ihnen zugewiesene Rolle als Gäste verlassen, die sie in den 1960er und 1970er Jahren auch räumlich in das innere Ausland (Türkenschulen/ Wohnvierteln in randständigen Bezirken oder Hinterhofmoscheen etc.) verwies, die eine Nicht-Beachtung - im Sinne einer "Vergegnung" - ermöglichte. Wurden sie (auch im eigenen Selbstverständnis) in den ersten zwanzig Jahren der Einwanderung in der Rolle als „Gast“-Arbeiter primär als Hilfsarbeiter wahrgenommen, die Arbeiten zu verrichten hatten, für die nicht genügend Einheimische zur Verfügung standen, um nach verrichteter Arbeit in die Heimatländer zurückzukehren, so haben sich diese Rückkehr-Erwartungen beiderseits nicht erfüllt. Sie sind heute in der Mitte der Gesellschaft angekommen. Allen ist nun klar: Die ehemaligen Gastarbeiter haben ihren Lebensmittelpunkt auf Dauer in die Bundesrepublik verlegt und beanspruchen, „Objekt von Verantwortung“ (Bauman) zu sein. Als mündige Bürger dieses Landes beanspruchen sie Rechte und Rollen, die für sie nicht vorgesehen waren. Die Nachkommen der ursprünglich zugewanderten „Gast"-Arbeiter mit entsprechenden Bildungsabschlüssen konkurrieren 
nun nicht mehr nur mit dem Hilfsarbeiter, sondern mit Angestellten und Beamten. Es bedurfte nun einer Neujustierung der Rollen innerhalb des Gesellschaftsgefüges, die die Marginalisierung der mittlerweile etablierten Einwanderer weiterhin zu gewährleisten hatte. Im Zuge dessen ist der Islam zum Gegenstand öffentlicher Anerkennungskonflikte geworden. D.h. in der Konkurrenz um die besten Plätze innerhalb der Gesellschaft werden antiislamische Vorurteile und Feindbilder als Ausschließungspraxis eingesetzt, da das Privileg des „Staatsbürgers für die autochthone Gruppe“ nicht mehr greift. Daher sind islamfeindliche Haltungen und Tendenzen mittlerweile nicht nur am (rechten) Rand der Gesellschaft zu finden, sondern auch in der Mitte, wie wir dies am Erfolg der AfD (Alternative für Deutschland) sehen, die sich als parlamentarischer Arm des rechtspopulistischen Spektrums etabliert hat.

\section{AFD - DIE „PROTEST-PARTEI““ GEGEN DEN ISLAM}

Mittlerweile hat sich eine im Jahre 2013 gegründete Partei Alternative für Deutschland (AfD) mit offen geäußerten islamfeindlichen Positionen neben den etablierten Volksparteien mit besorgniserregender anwachsender Popularität im Parteienspektrum etabliert. ${ }^{11}$ Nach einem monatelangen innerparteilichen Machtkampf im Jahre 2015, der schließlich zum Ausschluss bzw. Austritt ursprünglicher $A f D$-Begründer führte, wurde diese ursprünglich gegen die Europapolitik gerichtete Bewegung immer mehr zu einer rechtspopulistischen Anti-Islam-Partei, für die der Islam eine dem Grundgesetz widersprechende politische Ideologie ist. So ist für den ehemaligen AfD-Chef Alexander Gauland der Islam „keine Religion wie das katholische oder protestantische Christentum, sondern intellektuell immer mit der Übernahme des Staates verbunden. "12 Für ihn gibt es neben der fundamentalen Ausrichtung des Islam keinen aufgeklärten Islam, der mit der freiheitlich-demokratischen Grundordnung vereinbar sei. ${ }^{13}$ Noch wesentlich schärfer formulierte dies der ehemalige stellvertretende Chefredakteur der „Bild am Sonntag“ Nicolaus Fest, ehemalige Sprecher, der vom Januar 2020 bis März 2021 Vorsitzender des Notvorstands der Berlin AfD Berlin war. Er sprach sich 2016 dafür aus, dass für Deutschland keine andere Möglichkeit bestünde, als „alle Moscheen zu schließen. “ ${ }^{14}$ Anlässlich

${ }^{11}$ Nach Erhebungen des Deutschland Trend von Infratest vom 21.9. 2018 schob sich die AfD erstmals an der SPD auf den zweiten Platz vorbei (vgl. https://www.tagesschau.de/inland/deutschlandtrend1381.html) (Zugriff am 5.10 2018).

${ }^{12}$ Deutschlandfunk, „Der Islam ist ein Fremdkörper“(Zugriff am 11. 10 2018).

${ }^{13}$ vgl. FAZ-Sonntagszeitung, „Gauland: Verfasster Islam nicht mit Grundgesetz vereinbar“ (Zugriff am 3. 9. 2019)

${ }^{14}$ Markus Decker, „Prominentes neues AfD-Mitglied“. Frankfurter Rundschau (Oktober 2016), 72.Jg., 
seiner offiziellen Vorstellung als neues Parteimitglied hob Fest hervor, dass der Islam eine „.. eine totalitäre Bewegung“, sei. ${ }^{15}$

Die AfD fixierte schließlich anlässlich ihres Bundesparteitages vom 30.4./1.5. 2016 ihre Anti-Islam-Rhetorik in ihrem Grundsatzprogramm. Dort wurde mit dem Unterpunkt 7.6.1 programmatisch festgehalten: „Der Islam gehört nicht zu Deutschland“. ${ }^{16}$ Als Bundestagsfraktion brachte die AfD am 11.10. 2018 schließlich einen Antrag „Unvereinbarkeit von Islam, Scharia und Rechtsstaat - Der Radikalisierung den Boden entziehen, keine Verbreitung gesetzwidriger Lehren" ein, womit die Bundesregierung aufgefordert wurde, die Verbreitung von im Koran enthaltenen gesetzwidrigen Inhalten und Aufrufen zu unterbinden, dem seitens der übrigen Fraktionen widersprochen wurde. ${ }^{17}$

Inzwischen kam es zu einem ideologischen Zusammenschluss von AfD mit dem Bündnis „Patriotische Europäer gegen die Islamisierung des Abendlandes" (Pegida). Mit einem AfD-Parteibeschlusses 2018 wurde es AfDMitgliedern zukünftig gestattet, gemeinsam mit der Pegida zu demonstrieren, womit das 2016 beschlossene Kooperationsverbot der AfD mit dem ausländerfeindlichen Pegida-Bündnis zusammenzuarbeiten aufgehoben wurde $^{18}$ Inzwischen sammelte sich eine Gruppe völkisch-nationalistischer, rechtsextremer Parteigenossen um den Thüringer AfD-Vorsitzenden Hökke, die unter dem Namen „Der Flügel“ der AfD fungierte, dem neben anderen auch der Islamwissenschaftler und Landtagsabgeordneter von SachsenAnhalt, Hans-Thomas Tillschneider, angehörte. Dieser Zusammenschluss rechtsextremistischer, islamfeindlicher Kräfte der AfD, die sich insbesondere gegen gemäßigte Strömungen der eigenen Partei richtete, wurde allerdings im Jahre 2020 wegen rechtsextremistischer Bestrebungen durch den Verfassungsschutz nachrichtendienstlich beobachtet und deshalb seitens der AfD offiziell als aufgelöst erklärt.

Als weiteres Bindeglied zwischen AfD und PEGIDA kann wohl die seit dem Beginn der 2016er Jahre zunehmend ins Blickfeld geratene rechtsnational-völkisch orientierte Bewegung der „Identitären“"19, die sich als Teil der „Neuen Rechten“ ${ }^{\text {"20 }}$ bezeichnet, angesehen werden. Die „Identitären“

\footnotetext{
Nr.233, 5.; Ferdinand Otto, „Ein neuer Scharfmacher für die AfD“. Die Zeit (Zugriff am 11. 10 2018).

${ }^{15}$ Focus Online, „Neuer AfD-Mann vergleicht Islam mit Nationalsozialismus“ (Zugriff am 3. 4. 2018)

16 Alternative für Deutschland (AfD), „Programm für Deutschland. Das Grundsatzprogramm der AfD“ (Zugriff am 19.12.2021)

17 Deutscher Bundestag, „AfD-Antrag über Islam und Scharia erntet Widerspruch übriger Fraktionen“ (Zugriff am 20.12. 2018).

18 Zeit Online, „AfD Politiker dürfen bei Pegida auftreten“ (Zugriff am 11.8.2018)

19 https://www.identitaere-bewegung.de/ (Zugriff: 8.10. 2018).

${ }^{20}$ Richard Stöss, „Die ,Neue Rechte in der Bundesrepublik“ (Zugriff am 12.10.2018).
} 
$(I D)^{{ }^{\prime 21}}$, die sich als metapolitischer und aktivistischer Arm der Neuen Rechten in Abgrenzung zur Alten Rechten (Nationalisten, Rassisten, Neonazis etc.) verstehen und sich durch eine zunehmende Islamisierung und eines befürchteten „großen Austauschs“ die europäisch-kulturelle „Identi-

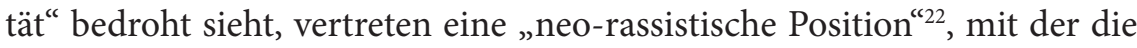
Achtung einer jeden Ethnie und Kultur betont wird, die sich und ihre identifikatorischen Besonderheiten im Sinne eines „Ethnopluralismus“ jedoch lediglich in ihrem angestammten Herkunftsgebiet zu entfalten und zu bewahren habe $e^{23}$

$\mathrm{Zu}$ diesem genannten islamfeindlichen Spektrum kommt eine kaum zu überblickende Vielzahl von offen geäußerten islamfeindlichen Positionen im Internet hinzu, die sich in bekannten Internetforen wie „Politically Incorrect" $\left.^{\text {" }} \mathrm{PI}\right)^{24}$ finden lassen, in denen, wie es Gerhold nennt, zum „Islambashing für jedermann" eingeladen wird ${ }^{25}$

Es muss in diesem Zusammenhang auch eingeräumt werden, dass nicht alle kritischen Haltungen zum Islam von einem antiislamischen Ethnizismus mit entsprechendem Hasspotenzial zeugen. Ein nicht unerheblicher Teil, die beispielsweise bei (Pegida) mitlaufen, sind Menschen, die im Zuge eines auf den Islam bezogenen Ethnizismus Ängsten und Vorurteilen aufsitzen, aber wohl mehrheitlich (noch) nicht von einer Ideologie der Ungleichwertigkeit oder von einem geschlossenen Feindbild gegenüber dem Islam durchdrungen sind. Hier bleibt zu hoffen, dass dieser Teil politischen Konzepten und vernünftigen Argumenten noch zugänglich ist und deshalb gesellschaftspolitischer Aufmerksamkeit bedarf.

\section{FAZIT}

In diesem Artikel wurde ausgeführt, dass die moderne Form der Islamfeindlichkeit auch eine Reaktion auf die Dauerpräsenz von Muslim_innen im Zuge der Einwanderungsgeschichte in Deutschland und die damit verbundene Irritation der Rollen zwischen dem Gast und dem Gastgeber ist.

${ }^{21}$ www.identitaere-bewegung.de/idee \& tat/ (Zugriff: 26.09.2016).

${ }^{22}$ Mit „Neo-Rassismus“ ist ein „Rassismus ohne Rassen“ gemeint, der gegenüber dem klassisch-biologischen Rassismus andere Begründungen heranzieht, um Ungleichwertigkeiten zwischen Menschen-(Gruppen) zu begründen und Hierarchien zu etablieren. Gegenüber früheren biologischen Rassemerkmalen sind es dort die betonten unvereinbaren Lebensweisen unterschiedlicher Kulturen bzw. Ethnien, wobei hier im Grunde der Terminus „Ethnie“ gewissermaßen als euphemistischer Ersatz für den kompromittierenden Begriff „Rasse“ steht.

${ }^{23}$ vgl. Andreas Speit, Das Netzwerk der Identitären. Ideologie Aktionen der Neuen Rechten (Berlin: Christian Links Verlag, 2018), 10.

${ }^{24}$ Die PI-Website ist einsehbar unter http://www.pi-news.net.

${ }_{25}$ Markus Gerhold, „Islam-bashing für jedermann. Leserbriefe und Onlinekommentare als Ort privater Stimmungsmache“, In: Thorsten Gerald Schneiders (Hrsg.), Islamfeindlichkeit, Wenn die Grenzen der Kritikverschwimmen (Wiesbaden: VS Verlag für Sozialwissenschaften, 2009), 231 
Hierbei werden antiislamische Vorurteile und Feindbilder als Mechanismen der sozialen Ausgrenzung gegenüber Migrant_innen aus muslimisch geprägten Herkunftsstaaten oder Menschen, die als Muslime markiert werden, aufgegriffen bzw. reproduziert, um insbesondere diejenigen, die bereits in der Mitte der Gesellschaft angekommen sind, an die randständigen Positionen innerhalb der Gesellschaft zu binden.

Genannt sei hier zudem als markanter Punkt, die der modernen Islamfeindlichkeit in den 1990er Jahren Anschub verlieh, das Ende des „Kalten Krieges und dem damit verbundenen Ende der poststalinistisch-osteuropäischen Systeme. Seither kam dem Islam anstelle des Antikommunismus' eine zentrale Feindbildfunktion zu, die offenbar zum Zwecke der Selbstdefinition und Selbstvergewisserung als dialektisches Gegenüber zur eigenen Identitätserfassung existenziell notwendig war und ist. Nicht zu vernachlässigen ist ebenfalls die deutsche Wiedervereinigung, die mit der Suche nach einer neuen deutschen Identität einherging. Dennoch kann die Islamfeindlichkeit nicht nur auf aktuelle Ereignisse der Zeitgeschichte reduziert werden.

\section{LITERATUR}

Attia, Iman. Die „westliche Kultur“ und ihr Anderes. Zur Destruktion von Orientalismus und antimuslimischem

Rassismus. Bielefeld: transcript, 2009.

Bielefeldt, Heiner. Das Islambild in Deutschland. Zum öffentlichen Umgang mit der Angst vor dem Islam. In: Schneiders, Th. G. (Hrsg.): Islamfeindlichkeit. Wenn die Grenzen der Kritik verschwimmen. Wiesbaden: VS-Verlag, 2009, 167-200.

Bielefeldt, Heiner. Facetten von Muslimfeindlichkeit. Differenzierung als Fairnessgebot. Überarbeitete Fassung eines Vortrags vom 6. September 2010; online unter: http://www.deutsche-islam konferenz.de/SharedDocs/Anlagen/DE/DIK/Downloads/Sonstiges/vortrag-bielefeldt,tem plateId $=$ raw, property $=$ publicationFile.pdf/vortrag-bielefeldt.pdf $(\mathrm{Zu}-$ griff 25.Oktober 2018).

Bühl, Achim. Islamfeindlichkeit in Deutschland. Hamburg: VSA, 2010.

Çakır, Naime. Islamfeindlichkeit - Anatomie eines Feindbildes in Deutschland. Bielefeld: Transcript, 2014.

Çakır, Naime. „Das Eigene und das Fremde - zwischen Heterophobie und Rassismus“. In: Uçar Bülent - Kassis

Wassilis (Hrsg.), Antimuslimischer Rassismus und Islamfeindlichkeit, Göttingen: Vandenhoeck \& Ruprecht, 2019, 77-101. 
Decker, Markus. „Prominentes neues AfD-Mitglied“. Frankfurter Rundschau 72.Jg., Nr.233, (Oktober 2016).

Gerhold, Markus. „Islam-bashing für jedermann. Leserbriefe und Onlinekommentare als Ort privater

Stimmungsmache“, In: Schneiders, Thorsten Gerald (Hrsg.): Islamfeindlichkeit. Wenn die Grenzen der Kritik

verschwimmen. Wiesbaden: VS Verlag für Sozialwissenschaften, 2009.

Hafez, Farid (Hrsg.), Jahrbuch für Islamophobieforschung, Wien: ZDB-Verlag 2010.

Heitmeyer, Wilhelm (Hrsg.). Deutsche Zustände. Folge 1. Frankfurt/Main: Suhrkamp, 2002.

Heitmeyer, Wilhelm (Hrsg.). Deutsche Zustände. Folge 2. Frankfurt/Main: Suhrkamp, 2003.

Heitmeyer, Wilhelm., „Gruppenbezogene Menschenfeindlichkeit - Die theoretische Konzeption und empirische Ergebnisse aus 2002, 2003 und 2004“. In: Heitmeyer, Wilhelm (Hrsg.), Deutsche Zustände. Folge 3. Frankfurt/Main 2005: Suhrkamp, 13-34.

Heitmeyer, Wilhelm (Hrsg.). Deutsche Zustände. Folge 6. Frankfurt/Main: Suhrkamp, 2008.

Schiffer, Sabine. „Islamophobie - Plädoyer für eine internationale Bezeichnung“. In. INAMO. Feindbild Islam. Islamfeindlichkeit und Rechtspopulismus. Heft 68, Jahrgang 17, Winter 2011, 22-24

Schneiders, Thorsten Gerald (Hrsg.). Islamfeindlichkeit. Wenn die Grenzen der Kritik verschwimmen. Wiesbaden: VS-Verlag, 2009.

Speit, Andreas (Hrsg.). Das Netzwerk der Identitären. Ideologie Aktionen der Neuen Rechten. Berlin: ChristianLinks Verlag, 2018.

\section{Zeitungs- und Zeitschriftenartikel (online):}

Deutschlandfunk. „Der Islam ist ein Fremdkörper“ (April 2016), online: https://www.deutschlandfunk.de/afd-der-islam-ist-ein-fremdkoerper-100.html (Zugriff: 11. 10 2018)

FAZ-Sonntagszeitung, „Gauland: Verfasster Islam nicht mit Grundgesetz vereinbar“ (Mai 2019), online: https://www.faz.net/aktuell/politik/ inland/afd-chef-gauland-verfasster-islam-nicht-mit-grundgesetzvereinbar-16171578.html (Zugriff: 3. 9. 2019).

Ferdinand Otto, „Ein neuer Scharfmacher für die AfD“. Die Zeit (Okober 2016), online: http://www.zeit.de/politik/2016-10/nicolaus-festjournalist-afd-beitritt-islam (Zugriff am 11. 10 2018). 
Focus Online, „Neuer AfD-Mann vergleicht Islam mit Nationalsozialismus“ (Oktober 2016), online: https://www.focus.de/politik/deutschland/ nicolaus-fest-neuer-afd-mann-vergleicht-islam-mit-nationalsozialismus_id_6038387.html (Zugriff 3.4. 2018).

Stöss, Richard „Die ,Neue Rechte 2016), online: http://www.bpb.de/politik/extremismus/ rechtsextremismus/229981/die-neue-rechte-in-der-bundesrepublik (Zugriff: 12.10.2018).

Zeit Online, „AfD Politiker dürfen bei Pegida auftreten“ (April 2018), online: https://www.zeit.de/news/2018-03/04/parteibeschluss-afdpolitiker-duerfen-bei-pegida-auftreten-180304-99-333607 (Zugriff: 11.8.2018). 